\title{
Patients Requiring Gastrostomy Tube Insertion After Total Laryngectomy Have a Higher Incidence of Otitis Media
}

\author{
Youngrak Jung, Ara Cho, Seungjoon Yang, Yutae Jeon, and Seong Hoon Bae \\ Department of Otorhinolaryngology, Yonsei University College of Medicine, Seoul, Korea
}

Received September 27, 2021

Revised November 2, 2021

Accepted November 24, 2021

\author{
Address for correspondence \\ Seong Hoon Bae, MD PhD \\ Department of Otorhinolaryngology, \\ Yonsei University \\ College of Medicine, \\ 50-1 Yonsei-ro, Seodaemun-gu, \\ Seoul 03722, Korea \\ Tel $+82-2-2228-3611$ \\ Fax +82-2-393-0580 \\ E-mail bshsap1@yuhs.ac
}

\begin{abstract}
Background and Objectives: Some reports propose an increased risk of otitis media and hearing impairment after total laryngectomy. However, the incidence of otitis media following laryngectomy and the mechanism remain unclear. This study aimed to identify the incidence and risk factors of otitis media after total laryngectomy. Subjects and Methods: This retrospective cohort study assessed 77 patients who underwent total laryngectomy from 2010 to 2020 in a tertiary referral center. Serial imaging studies (computed tomography [CT], magnetic resonance imaging, and positron emission tomography-CT) were used to assess otitis media. Results: The study enrolled 58 patients (mean age, $67.0 \pm 7.7$ years; male, 56 [96.6\%]); nine (15.5\%) underwent a gastrostomy tube (four preoperatively and five postoperatively). Otitis media was confirmed in seven (12.1\%) patients. Gastrostomy tube insertion was the only significant risk factor for otitis media $(p=0.012)$. Of the nine patients who underwent gastrostomy tube insertion, four developed otitis media; all four had the procedure after laryngectomy. Conclusions: This study found an increased incidence of otitis media after total laryngectomy. Swallowing difficulties likely contribute to otitis media as it occurred more frequently in patients requiring postoperative gastrostomy tube insertion.

J Audiol Otol 2022;26(1):31-35
\end{abstract}

\section{Introduction}

Many surgical techniques try to preserve the larynx during laryngeal cancer or other advanced head and neck cancer treatments, such as partial laryngectomy and robot-assisted surgery. Despite surgical advancement, total laryngectomy remains a major surgical procedure for head and neck malignancies. Total resection of the larynx leads to remarkable anatomical changes in the patient's head and neck by creating a discontinuity between the upper and lower airways and directly exposing the trachea. Communication problems from losing the voice are the most significant changes, but other issues include trachea dryness, crusting, and bleeding and common lower respiratory tract infections [1]. Further, nasal airflow interruptions lead to nasal mucosa modification, resulting in impaired nasal function [2].

This is an Open Access article distributed under the terms of the Creative Commons Attribution Non-Commercial License (https://creativecommons.org/licenses/by-nc/4.0/) which permits unrestricted non-commercial use, distribution, and reproduction in any medium, provided the original work is properly cited.
Along with these problems, an increased risk of otitis media and hearing impairment after total laryngectomy has also been proposed [3,4]. In addition to losing the voice, postoperative hearing impairment exacerbates communicative problems, posing difficulties in conveying critical information during the immediate postoperative period and the rehabilitation process [5]. Anatomically, several factors increase the prevalence of otitis media after total laryngectomy. For instance, nasal airflow exclusion, decreased swallowing function, esophageal reflux, and postoperative radiation therapy can affect the middle ear status [6-10]. However, the incidence of otitis media following laryngectomy is not well investigated and there have been no recent studies regarding its mechanism.

This study aimed to identify the otitis media incidence rate and risk factors after total laryngectomy. If vulnerable patient groups are identified in advance, then the patients can be closely monitored and appropriate interventions implemented, which is important for postoperative care and rehabilitation. 


\section{Subjects and Methods}

\section{Participants}

This study was approved by the ethics committee of Severance Hospital (approval number 4-2021-0814), and was a retrospective study that did not cause any harm to the study subjects; therefore, the requirement of informed consent was waived by the board. Medical records were reviewed from 2010 to 2020 to identify all patients who underwent total laryngectomy due to a head and neck malignancy; 77 patients were identified. The exclusion criteria were a lack of imaging preoperatively and six months postoperatively, preoperative history of an ear disorder (e.g., mastoidectomy, chronic otitis media, serous otitis media), and abnormal preoperative imaging findings in the mastoid cavity or middle ear. The preoperative imaging was implemented within 3 months before surgery. Based on these criteria, 19 patients were excluded, and 58 patients were enrolled. A retrospective chart review gathered the sex, age, radiation therapy history, esophageal and pharyngeal involvement in the resection margin, gastrostomy tube insertion, and esophagogram findings (pre and postoperative) for each patient. The esophagogram findings were evaluated on the basis of official readings.

Table 1. Demographic and clinical characteristics of enrolled patients

\begin{tabular}{lc}
\hline \multicolumn{1}{c}{ Factors } & \multicolumn{1}{c}{ Value } \\
\hline Age $(\mathrm{yr})$ & $67.0 \pm 7.7$ \\
Male sex & $56(96.6)$ \\
Radiation therapy & $35(60.3)$ \\
Gastrostomy tube & $9(15.5)$ \\
Pharynx involvement & $51(87.9)$ \\
Esophagus involvement & $18(31.0)$ \\
Abnormal esophagogram & $19(32.8)$ \\
Otitis media & $7(12.1)$ \\
Total number & $58(100)$ \\
\hline
\end{tabular}

Data are presented as mean \pm standard deviaton or $\mathrm{n}(\%)$

\section{Otitis media with effusion evaluation}

Pre- and postoperative serial imaging studies were assessed for otitis media. The imaging studies included computed tomography (CT), magnetic resonance imaging (MRI), and positron emission tomography (PET) scans. The imaging findings of otitis media were soft tissue density in CT or PET-CT scans and higher signal intensity than the turbinate mucosa or similar intensity to cerebrospinal fluid in T2-weighted MRI images [10]. These findings in the middle ear cavity, antrum, or nearby air cells confirmed otitis media.

\section{Statistical analyses}

Statistical analysis was performed using SPSS software version 25 for Windows (IBM Corp., Armonk, NY, USA). Age, male sex, radiation therapy history, gastrostomy tube insertion, abnormal esophagogram (anastomosis site leakage or fistula), pharynx involvement, and esophagus involvement were assessed as risk factors for otitis media by logistic regression analysis. A $p$-value less than 0.05 was considered statistically significant.

\section{Results}

\section{Patient demographics and otitis media incidence}

In total, 58 patients were enrolled (Table 1). The mean age at the time of surgery was 67 (range, 52 to 83 ) years; 56 were men $(96.6 \%)$. Of the 58 patients, 51 patients $(87.9 \%)$ underwent pharyngectomy (partial or total) with total laryngectomy, and $18(31.0 \%)$ also needed partial esophageal resection due to advanced disease. Nine patients $(15.5 \%)$ underwent gastrostomy tube insertion (four preoperatively and five postoperatively). Adjuvant radiation therapy was administered in 35 patients $(60.3 \%)$. Otitis media was confirmed in seven patients, and the otitis media incidence of 6 months postoperatively was $12.1 \%$.

\section{Otitis media risk factors after total laryngectomy}

Only gastrostomy tube insertion was a significant risk fac-

Table 2. Logistic regression analysis of otitis media risk factors

\begin{tabular}{llccc}
\hline \multicolumn{1}{c}{ Factors } & Value & Odds ratio & $p$-value & 95\% Confidence Interval \\
\hline Age & Continuous & 1.109 & 0.228 & $0.937-1.311$ \\
Male sex & Dichotomous & 0.000 & $>0.999$ & N/A \\
Radiation therapy & Dichotomous & 3.909 & 0.310 & $0.281-54.453$ \\
Gastrostomy tube & Dichotomous & 21.518 & $0.016^{*}$ & $1.759-263.302$ \\
Pharynx involvement & Dichotomous & 0.227 & 0.337 & $0.011-4.684$ \\
Esophagus involvement & Dichotomous & 0.174 & 0.283 & $0.007-4.243$ \\
Abnormal esophagogram & Dichotomous & 5.953 & 0.118 & $0.635-55.822$ \\
\hline
\end{tabular}

Model accuracy=93.1\%. Nagelkerke $R^{2}=0.514$. Hosmer \& Lemeshow=0.826. *statistically significant. N/A, not available 
tor for otitis media $(p=0.016$ ) (Table 2$)$. Of the nine patients who underwent gastrostomy tube insertion, four developed otitis media (Table 3). Interestingly, all underwent the procedure postoperatively, and three of the four suffered postoperative structural complications. These structural complications were confirmed by esophagography and the three patients accompanied anastomosis site leakage. The patients who underwent preoperative gastrostomy insertion did not develop otitis media postoperatively.

\section{Discussion}

In our study, $12.1 \%$ of patients ( 7 of 58 ) developed otitis media within six months after total laryngectomy. In 2005, the World Health Organization reported that, globally, $51 \%$ of otitis media cases occurred in patients aged $<5$ years, and the global incidence rate was between 1.49 and 3.14 cases per 100 people-years for people aged $\geq 20$. Given that otitis media in the general adult population is rare, the otitis media prevalence appears to increase after total laryngectomy $[11,12]$. In this study, gastrostomy tube insertion was significantly associated with otitis media, and $80 \%$ (4 of 5) of patients who underwent postoperative gastrostomy tube insertion developed otitis media. Considering that patients who underwent preoperative gastrostomy tube did not develop otitis media, it is likely that the predisposing condition prompting the tube insertion is a contributing factor.

Gastrostomy tube insertion was considered when a patient's dysphagia or oral food intake restriction was expected to be long-lasting $[13,14]$. Given that the patients with preoperative gastrostomy tube insertion were otitis media-free, the predisposing factors like structural problems related to the gastrostomy tube insertion and surgery may cause otitis media. For instance, anastomosis site leakage requires oral intake restriction and saliva secretion inhibition, possibly reducing the swallowing rate and effort. Although there was no statistical significance in regression analysis, 5 of 7 otitis media with effusion (OME) patients exhibit anastomosis site leakage or fistula in esophagography images.

To date, there is no direct evidence that dysphagia causes otitis media. However, a reduced swallowing rate and effort can mitigate ventilation of the middle ear via the Eustachian tube [7]. This finding is supported by Atsmoni et al. [15], who reported that oral feeding is associated with better Eustachian tube function and lesser middle ear problems in tracheotomy patients. Further, Chung et al. [16] reported significantly more improvement from otitis media after tracheotomy in conscious patients than in unconscious patients in the intensive care unit, which also supports the relationship between swallowing and

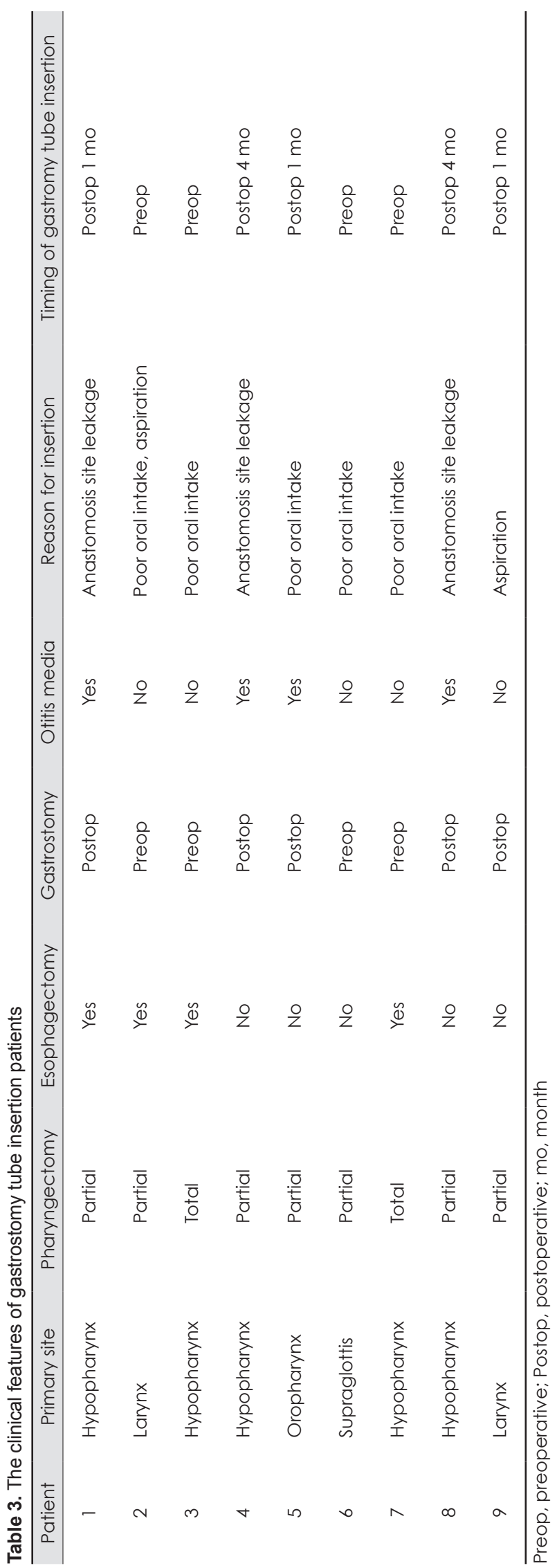

www.ejao.org 33 
Eustachian tube function. Additionally, swallowing difficulties may result in the poor clearing of liquid contents from the nasopharynx that can regurge into the middle ear via the Eustachian tube. Indeed, laryngopharyngeal reflux disease (LPRD) has been associated with otitis media [8]. Similarly, gastroesophageal reflux disease (GERD) has also been correlated with otitis media [17]. Together, gastrostomy tube insertion is likely not a direct cause of otitis media but rather the swallowing difficulty.

Several studies reported nasal cavity changes after total laryngectomy. Nasal airflow interruptions deprive the nasal cavity of its physiological functions, which is connected to increasing endonasal heat and humidity [18], decreased endonasal blood flow [19], and physical and histologic nasal mucosa changes. Although these changes can affect the middle ear, Meric et al. [20] reported that the Eustachian tube function was preserved after total laryngectomy. In our study, only $12.1 \%$ of patients had otitis media during the six-month postoperative follow-up period, a relatively short follow-up period compared to Meric et al. [20]. It is possible that the swallowing function could recover during a longer-term follow-up period. The short-term changes in Eustachian function after total laryngectomy should be investigated in future studies.

This study has several limitations. First, otitis media was only confirmed using by imaging study in 6 months after surgery. Other information, including clinical symptoms, physical exams, and tympanometry, was unavailable because of the retrospective review limitations. For this reason, there is also a possibility of misdiagnosed cases due to the lack of comprehensive and consecutive information. Second, more specific analyses regarding swallowing function are required. If an objective swallowing function measurement could be implemented, correlations between swallowing difficulty and otitis media could be identified. Third, other factors, including sinusitis, pneumonia, LPRD, or GERD, can be related to the OME development. However, in our cohort, only 2 of 7 patients had sinusitis and/or pneumonia in the perioperative period. Also, there were not available test results or medical records for evaluating reflux diseases. A larger cohort and more comprehensive data would be required to verify all possible factors that cause OME.

In conclusion, this retrospective study found an increased incidence otitis media rate in total laryngectomy patients. Swallowing difficulties likely contribute to otitis media as it occurred more frequently in patients requiring postoperative gastrostomy tube insertion.

\section{Acknowledgments}

This research was supported by the Basic Science Research Pro- gram through the National Research Foundation of Korea, funded by the Ministry of Education (NRF-2020R1I1A1A01067241 to SH Bae).

\section{Conflicts of interest}

The authors have no financial conflicts of interest.

\section{Author Contributions}

Conceptualization: Seong Hoon Bae. Data curation: Youngrak Jung, Seungjoon Yang, Ara Cho, Yutae Jeon. Formal analysis: Seong Hoon Bae. Methodology: Seong Hoon Bae, Youngrak Jung. Project administration: Seong Hoon Bae, Youngrak Jung. Writing — original draft: Youngrak Jung. Writing — review \& editing: Seong Hoon Bae. Approval of final manuscript: all authors.

\section{ORCID iDs}

Youngrak Jung

Ara Cho

Seungjoon Yang

Yutae Jeon

Seong Hoon Bae https://orcid.org/0000-0001-7893-1866 https://orcid.org/0000-0002-1863-4883 https://orcid.org/0000-0002-4468-3646 https://orcid.org/0000-0001-7718-4520 https://orcid.org/0000-0001-9243-9392

\section{REFERENCES}

1) Hilgers FJ, van Dam FS, Keyzers S, Koster MN, van As CJ, Muller MJ. Rehabilitation of olfaction after laryngectomy by means of a nasal airflow-inducing maneuver: the "polite yawning" technique. Arch Otolaryngol Head Neck Surg 2000;126:726-32.

2) Cvetnić V, Skrlin J, Rak I. Bacterial flora of the nasal cavity in laryngectomized patients. Infection 1996;24:26-8.

3) Cameron P, Green WB, Gulliver M. [Middle ear dysfunction following laryngectomy]. Can J Speech Lang Pathol Audiol 2000;24: 19-25.

4) Berlin CI. Clinical measurement of esophageal speech. I. Methodology and curves of skill acquisition. J Speech Hear Disord 1963;28:4251 .

5) Doyle PC. Foundations of voice and speech rehabilitation following laryngeal cancer. San Diego: Singular Publishing Group;1994.

6) Watson C. Chronic otitis media: the significance of nasal obstruction. Clin Otolaryngol Allied Sci 1990;15:435-8.

7) Tysome JR, Sudhoff H. The role of the Eustachian tube in middle ear disease. Adv Otorhinolaryngol 2018;81:146-52.

8) Karyanta M, Satrowiyoto S, Wulandari DP. Prevalence ratio of otitis media with effusion in laryngopharyngeal reflux. Int J Otolaryngol 2019;2019:7460891.

9) Yüksel F, Doğan M, Karataş D, Yüce S, Şentürk M, Külahli I. Gastroesophageal reflux disease in children with chronic otitis media with effusion. J Craniofac Surg 2013;24:380-3.

10) Na G, Kim KH, Byun HK, Bae SH. Assessment of radiation-induced otitis media in patients with parotid gland malignancy. Acta Otolaryngol 2021;141:466-70.

11) Pontefract B, Nevers M, Fleming-Dutra KE, Hersh A, Samore M, Madaras-Kelly K. Diagnosis and antibiotic management of otitis media and otitis externa in United States veterans. Open Forum Infect Dis 2019;6:ofz432.

12) Monasta L, Ronfani L, Marchetti F, Montico M, Vecchi Brumatti L, Bavcar A, et al. Burden of disease caused by otitis media: systematic review and global estimates. PLoS One 2012;7:e36226.

13) Yanni A, Dequanter D, Lechien JR, Loeb I, Rodriguez A, Javadian $\mathrm{R}$, et al. Malnutrition in head and neck cancer patients: impacts and indications of a prophylactic percutaneous endoscopic gastrostomy. Eur Ann Otorhinolaryngol Head Neck Dis 2019;136:S27-33.

14) Löser C, Aschl G, Hébuterne X, Mathus-Vliegen EM, Muscaritoli 
M, Niv Y, et al. ESPEN guidelines on artificial enteral nutrition--percutaneous endoscopic gastrostomy (PEG). Clin Nutr 2005;24:84861.

15) Atsmoni SC, Weinberger H, Shummer E, Sakat R, Adelman C, Fraenkel ND, et al. The prevalence of otitis media with effusion in children with tracheotomy. Am J Otolaryngol Head Neck Surg 2019;2:1068.

16) Chung HK, Lin CC, Wang CY, Lin CD, Tsai MH, Chang CS. Improvement in otitis media with effusion in patients undergoing tracheostomy after prolonged endotracheal intubation. J Otolaryngol Head Neck Surg 2009;38:532-6.
17) Wu ZH, Tang Y, Niu X, Sun HY, Chen X. The relationship between otitis media with effusion and gastroesophageal reflux disease: a meta-analysis. Otol Neurotol 2021;42:e245-53

18) Sakakura Y, Ukai K, Majima Y, Murai S, Harada T, Miyoshi Y. Nasal mucociliary clearance under various conditions. Acta Otolaryngol 1983;96:167-73.

19) Bende M. Blood flow in human nasal mucosa after total laryngectomy. Acta Otolaryngol 1983;96:529-31.

20) Meric A, Dogan R, Kahya V, Eren SB, Yilmaz F, Ozturan O. Eustachian tube function following total laryngectomy. J Craniofac Surg 2012;23:e502-5. 